\title{
Dynamic control of the operation regimes of a mode- locked fiber laser based on intracavity polarizing fibers: experimental and theoretical validation
}

\author{
Guillermo E. Villanueva ${ }^{1, *}$ and Pere Pérez-Millán ${ }^{2}$ \\ ${ }^{1}$ Nanophotonics Technology Center, Universidad Politécnica de Valencia, Camino de Vera s/n, 46022, Valencia, Spain \\ ${ }^{2}$ Departamento de Física Aplicada-ICMUV, Universidad de Valencia, Dr. Moliner 50, 46100, Burjassot, Spain \\ ${ }^{*}$ Corresponding author: guivilib@ntc.upv.es
}

Received March 8, 2012; revised March 28, 2012; accepted April 16, 2012; posted April 17, 2012 (Doc. ID 164370); published May 29, 2012

\begin{abstract}
An intracavity polarizing fiber is proposed to control the emission regime of a passively mode-locked fiber laser. Stable operation in self-starting high and low dispersion soliton mode-locking and $100 \mathrm{GHz}$ multiwavelength regimes is demonstrated through numerical simulations and experimental validation. Mode-locking stability is ensured by a saturable absorber in the ring cavity. The effective selection of operation regime is dynamically carried out by controlling the intracavity polarization state. (c) 2012 Optical Society of America

OCIS codes: $\quad 060.3510,060.5530,140.4050$.
\end{abstract}

Mode-locked fiber lasers have proven to be suitable sources of ultrashort optical pulses for applications like ultrahigh-bit-rate communications, ultrafast spectroscopy, and biomedicine. Fiber lasers fully exploit the advantages of optical fibers, such as compact waveguiding, large gain, effective heat dissipation, and low-cost components [1]. When implementing passive mode-locking, pulse shaping is not limited by the speed of external electronic signal generators in comparison to active modelocking, allowing stable subpicosecond pulses [2]. A well-established technique for passive mode-locking is the use of fast saturable absorbers (SA), allowing singlepolarization self-started soliton mode-locking [3, 4 ]. On the other hand, Lyot filters based on the combination of in-line polarizers and birefringent fibers have been exploited for the generation of a multiwavelength emission regime [5]. Recently, polarizing (PZ) fibers with very low losses and ready to be spliced to standard fibers have become commercially available, and have been employed for nonlinear polarization rotation (NLPR) modelocking in fiber lasers [6]. In this Letter, we demonstrate that by introducing a PZ fiber in the cavity of an SA modelocked laser, pulsed soliton mode-locking with controllable cavity dispersion and multiwavelength emission regimes can be obtained by controlling the state of polarization at the input and output of the PZ fiber. Simulation and experimental validation of the proposed structure demonstrates the dynamic control of the three operating regimes. In comparison with other laser structures incorporating high-birefringent fibers and polarizers to implement intracavity Lyot filters [5], our proposal performs the polarizing effect in a fiber and does not require a polarizer.

The cavity of the fiber laser is shown in Fig. 1. A saturable absorber performs the mode-locking stabilization mechanism. The fiber laser, without the PZ fiber, operates in a solitonic mode-locked regime. When inserting the PZ fiber in combination with two polarization controllers, it is possible to control the polarization-dependent losses applied to the optical pulse each roundtrip. Together with its inherent high birefringence, the PZ fiber can partially implement an intracavity multiwavelength
Lyot filter [7] with configurability via the polarization controllers. The filtering effect of the PZ fiber results in a multiwavelength emission, therefore the fiber laser can be dynamically adjusted to operate in a pulsed or in a multiwavelength regime. Furthermore, this kind of single-polarization fiber exhibits different chromatic dispersion in each principal axis, known as birefringence dispersion [8]. The PZ fiber birefringence dispersion provides a tool to control the roundtrip accumulated dispersion of the oscillating optical signal. Adjusting the cavity dispersion budget allows the control of the solitonic mode-locking operation.

In order to numerically model the fiber laser emission performance, pulse propagation is computed through the nonlinear Schrödinger equation (NLSE). As polarization of the optical pulse has to be taken into account, then the coupled NLS equations (CNLSE) will be considered [9]. These are given by

$$
\begin{aligned}
& \left(\frac{\partial}{\partial z}+\beta_{1 x} \frac{\partial}{\partial t}+i \frac{\beta_{2 x}}{2} \frac{\partial^{2}}{\partial t^{2}}+\frac{\alpha_{x}}{2}-\frac{g}{2}-\frac{D_{g}}{2} \frac{\partial^{2}}{\partial t^{2}}\right) A_{x} \\
& \quad=i \gamma\left(\left|A_{x}\right|^{2}+\frac{2}{3}\left|A_{y}\right|^{2}\right) A_{x}+i \frac{\gamma}{3} A_{x}^{*} A_{y}^{2} \exp \left(-2 i \Delta \beta_{0} z\right),
\end{aligned}
$$

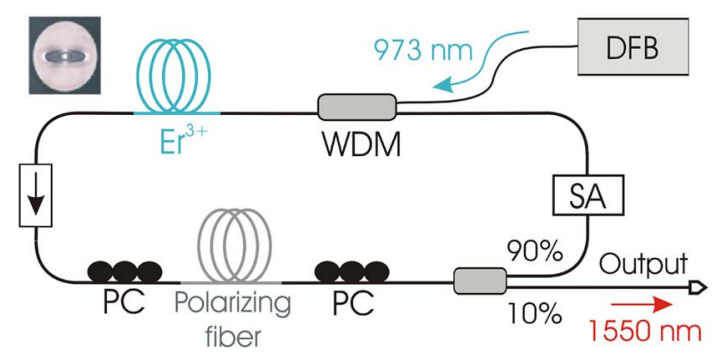

Fig. 1. (Color online) Scheme of the passively mode-locked fiber laser setup. DFB, distributed feedback pump diode laser; WDM, 980/1550 wavelength division multiplexer; $\mathrm{Er}^{3+}$, erbiumdoped fiber; PC, polarization controller; and SA, semiconductor saturable absorber. Inset: cross section of PZ fiber. 


$$
\begin{aligned}
& \left(\frac{\partial}{\partial z}+\beta_{1 y} \frac{\partial}{\partial t}+i \frac{\beta_{2 y}}{2} \frac{\partial^{2}}{\partial t^{2}}+\frac{\alpha_{y}}{2}-\frac{g}{2}-\frac{D_{g}}{2} \frac{\partial^{2}}{\partial t^{2}}\right) A_{y} \\
& \quad=i \gamma\left(\left|A_{y}\right|^{2}+\frac{2}{3}\left|A_{x}\right|^{2}\right) A_{y}+i \frac{\gamma}{3} A_{y}^{*} A_{x}^{2} \exp \left(2 i \Delta \beta_{0} z\right)
\end{aligned}
$$

Equations (1) and (2) describe the propagation of the slow-varying envelopes $A_{x}$ and $A_{y}$ for both linear polarizations. Birefringence of the optical fiber is denoted by the modal birefringence $\Delta \beta_{0}=\beta_{0 x}-\beta_{0 y}$, group birefringence $\beta_{1 i}$, and dispersion birefringence $\beta_{2 i}$, for $i=x, y$. Parameters $\alpha_{i}$ and $g$ denote the attenuation and gain of the optical fiber, respectively. The bandwidth of the gain medium is modeled by the gain dispersion $D_{g}$ through the relation $D_{g}=g / \Omega_{g}{ }^{2}$, where $\Omega_{g}$ is the HWHM gain bandwidth, assuming a parabolic approximation. Note the explicit polarization dependence of optical losses $\alpha_{i}$ and chromatic dispersion $\beta_{2 i}$, aimed to properly model the PZ fiber of the cavity. Nonlinear terms are governed by the fiber nonlinear coefficient $\gamma$. In Eqs. (1) and (2), more complex nonlinear effects, such as stimulated Raman scattering and self-steepening, are neglected. The CNLSE are solved using the split-step Fourier transform method.

The fiber ring cavity is composed of the following components: $3 \mathrm{~m}$ of erbium doped fiber Fibercore DHB1500 (peak absorption of $15 \mathrm{~dB} / \mathrm{m}$ at $1530 \mathrm{~nm}$ ) pumped by a $400 \mathrm{~mW}$ diode laser at $976 \mathrm{~nm}$ through a $980 / 1550 \mathrm{~nm}$ wavelength division multiplexer (WDM); $3 \mathrm{~m}$ of Verrillon highly birefringent polarizing fiber (birefringence $\Delta n=$ $10^{-3}$ and $0.6 \mathrm{~dB} / \mathrm{m}$ extinction ratio); a Batop fiber pigtailed fast semiconductor saturable absorber (relaxation time $\sim 2 \mathrm{ps}$, saturation energy $235 \mathrm{pJ}$, and modulation depth 15\%); an optical isolator to prevent bidirectional lasing operation; two polarization controllers (PCs) beside the polarizing fiber; a 90:10 output coupler; and finally $\sim 8 \mathrm{~m}$ of single-mode fiber (SMF) from all component pigtails. The dispersion of SMF and Er dopedfiber were -20 and $15 \mathrm{ps}^{2} / \mathrm{km}$, respectively, and the estimated $x$ and $y$ polarization dispersions of the PZ fiber were 20 and $53 \mathrm{ps}^{2} / \mathrm{km}$. A nonlinear coefficient of $1.5 \mathrm{mrad} /(\mathrm{W} \cdot \mathrm{m})$ is assumed. The cross section of the PZ fiber is shown in the inset of Fig. 1, where a highly elliptical cladding can be observed.

Optical pulses are propagated through fiber sections solving the CNLSE, and the PCs and SA effects are applied discretely. Each PC is modeled by three waveplates $(\lambda / 4-\lambda / 2-\lambda / 4)$ through their Jones matrix. In each simulation, an initial 1 ps Gaussian pulse is injected, and the output is extracted after convergence (usually reached in $\sim 1000$ roundtrips).

The PZ fiber is considered to be a Lyot filter in which the polarizing axis is identical to the birefringence axis, ideally preventing spectral filtering. In contrast to a conventional Lyot filter where an ideal polarizer is required, the PZ fiber shows an intended finite polarization extinction rate. Then a PZ fiber section can be analyzed as a high-birefringence fiber and a finite-extinction-ratio polarizer. If we include a polarization controller next to the PZ fiber, a spectral filtering can be deduced from the $x$ polarization components power transmission, given by

$$
\left|T_{x}\right|^{2}=\cos ^{2}(\theta)+\tau^{2} \sin ^{2}(\theta)+\tau \sin (2 \theta) \cos (\Delta \varphi),
$$

where $\Delta \varphi$ denotes the linear phase shift between two polarization components induced by the fiber, $\theta$ is the rotation angle between components introduced by the polarization controller, and $\tau$ are the polarizing losses. The operating regimes of the laser depend on the relative orientation of the light's state of polarization with regard to the polarization axis of the PZ fiber. The polarization state is adjusted by the polarization controllers. An orientation resulting in a low wavelength filtering results in a ineffective suppression of stimulation of the filtered wavelengths, hence the laser operates in a low dispersion solitonic regime with a repetition rate corresponding to the original FSR of the ring cavity $(\sim 15 \mathrm{MHz})$ and with a pulse width of 25 ps estimated from oscilloscope trace (see Fig. 4). Due to the unexpectedly high timebandwidth product, multipulse bunching is likely to occur, but the $7 \mathrm{ps}$ oscilloscope resolution was not sufficient to identify multipulse emission. Simulations provide pulse widths of 580 and 630 fs for the high and low dispersion soliton regime, respectively, and pulses energies of 100 pJ. Figures 2(a) and 2(b) shows the calculated and measured optical output spectrum, respectively, showing a bandwidth of $8.2 \mathrm{~nm}$ FWHM. Depending on the effective amount of polarization dispersion induced in the $\mathrm{PZ}$ fiber by the orientation of the PCs, a larger net anomalous dispersion can be obtained, and the laser works under a high dispersion soliton regime. The calculated average cavity dispersion for the low- and highdispersion regimes were -3.71 and $-0.073 \mathrm{ps}^{2} / \mathrm{km}$, respectively, and the measured temporal pulse width in this regime is $0.6 \mathrm{ps}$ (obtained from autocorrelation trace of the Fig. 4 inset). The differences in the Kelly sidebands
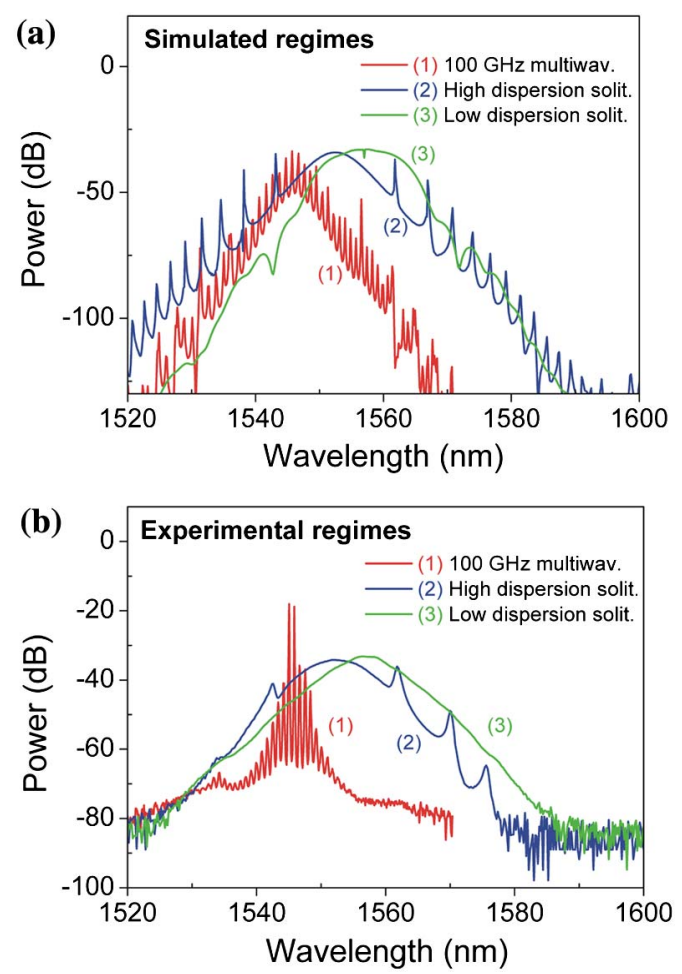

Fig. 2. (Color online) (a) Calculated and (b) measured optical output spectra of the three operation regimes of the laser. 


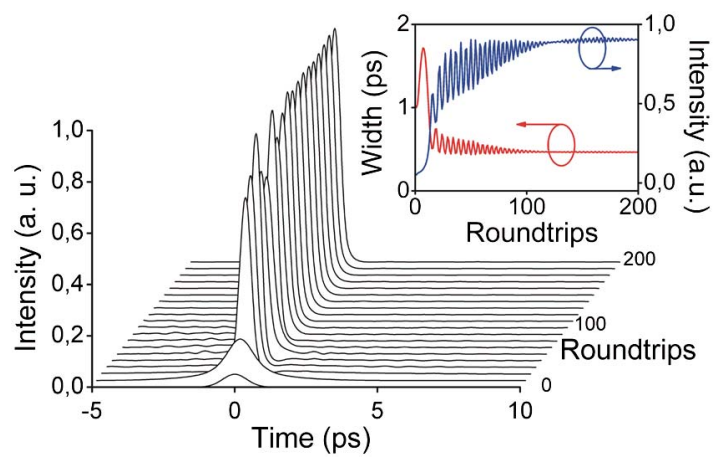

Fig. 3. (Color online) Calculated stable mode-locked pulse formation in the high dispersion solitonic regime. A Gaussian pulse of $1 \mathrm{ps}$ width is employed as an initial wavefunction. Inset: pulse width and peak intensity evolution.

observed in the calculated and simulated spectra in Fig. 2 are attributed to deviations in estimated cavity length and dispersion. When switching from a high-dispersion to a low-dispersion soliton regime, Kelly sidebands are no longer visible, as their spectral positions shift away from the central wavelength due to the low net dispersion [10]. Due to the polarizing effect of the PZ fiber, pulse shaping through NLPR can be expected, presenting a hybrid mode-locking mechanism [11] with an adjustable NLPR mechanism through polarization control. We could not mode-lock the laser without the SA for the same pumping and polarizing conditions. From the literature, NLPR is generally more unstable than SA mode-locking, and our laser shows good pulse stability, so we believe SA is the predominant mode-locking mechanism. Figure 3 shows a simulated high dispersion solitonic pulse shaping for an initial 1 ps width Gaussian pulse, reaching a 580 fs width steady-state in $\sim 200$ roundtrips.

The third operation regime is achieved when an orientation results in a high extinction ratio wavelength filtering, yielding a multiwavelength emission with an FSR of $106 \mathrm{GHz}$. The $106 \mathrm{GHz}$ wavelength spacing is in accordance with the length and birefringence of the PZ fiber following the relation

$$
\Delta \lambda=\lambda^{2} /\left(\Delta n L_{\mathrm{PZ}}\right),
$$

where $\Delta n$ and $L_{\mathrm{PZ}}$ denote the modal birefringence and length of the PZ fiber, respectively. Figure 2(b) shows the experimental optical output spectra for the three operation regimes. Each regime is achieved by fine adjustment of the polarization through the two PCs. For the two mode-locked regimes, a stable pulsed output signal is obtained. In contrast to $\mathrm{CW}$ multiwavelength lasers, in which NLPR can be exploited as gain equalization [5], the multiwavelength regime constitutes a harmonic mode-locking of $106 \mathrm{GHz}$ pulse repetition frequency. Unfortunately, no stable pulse emission was achieved from this regime. We believe spectral filter finesse and pumping power are not high enough to reach stable harmonic mode-locking. Figure 2(a) includes the three operation regimes of the simulated spectra. Figure $\underline{4}$ shows the temporal wavefunctions for both pulsed regimes.

In conclusion, a fiber laser with dynamic control of its operation regime has been proposed. High- and

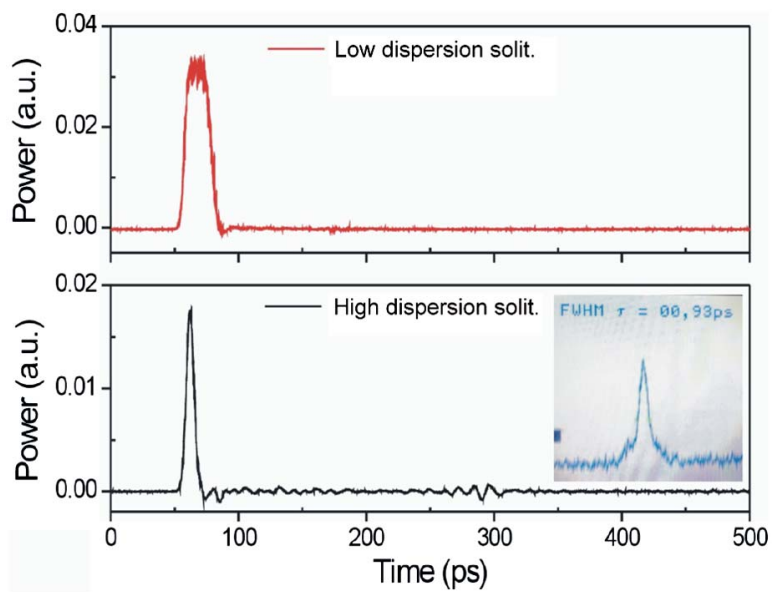

Fig. 4. (Color online) Pulsed temporal wavefunction of the laser output in the low-dispersion soliton regime and in the highdispersion soliton regime with an oscilloscope resolution of 7 ps. Inset: autocorrelation trace of the high-dispersion soliton regime pulses with a resolution of $10 \mathrm{fs}$.

low-dispersion soliton mode-locking, in addition to $106 \mathrm{GHz}$ multiwavelength regimes, are demostrated by a single cavity, and the dynamic selection of a particular working regime is performed via polarization control of the oscillating signal within the cavity. The operation control is based on a section of polarizing fiber inserted in the ring cavity, providing polarizing properties in addition to a high modal, group and dispersion birefringence. Effective regime control is demonstrated and evaluated experimentally and computionally. Pulse widths of $600 \mathrm{fs}$ and wavelength spacing of $106 \mathrm{GHz}$ for multiwavelength and solitonic operation, respectively, are obtained, finding potential application in high-bit-rate communications.

This work was financially supported by the European Commission under the FP7 EURO-FOS NoE (ICT-2007-2224402). The work of G. E. Villanueva was supported by the MEC-FPU programs. The work of P. Pérez-Millán was supported by the Juan de la Cierva program, JCI-200905805.

\section{References}

1. M. E. Fermann and I. Hartl, IEEE J. Sel. Top. Quantum Electron. 15, 191 (2009).

2. E. P. Ippen, Appl. Phys. B 58, 159 (1994).

3. H. Haus, IEEE J. Quantum Electron. 11, 736 (1975).

4. F. X. Kärtner, J. Aus der Au, and U. Keller, IEEE J. Sel. Top. Quantum Electron. 4, 159 (1998).

5. Z. Zhang, L. Zhan, K. Xu, J. Wu, Y. Xia, and J. Lin, Opt. Lett. 33, 324 (2008).

6. S. Li, X. Chen, D. Kuksenkov, J. Koh, M. Li, L. Zenteno, and D. Nolan, Opt. Express 14, 6098 (2006).

7. P. S. Liang, Z. X. Zhang, Q. Q. Kuang, and M. H. Sang, Laser Phys. 19, 2124 (2009).

8. D. Marcuse, IEEE J. Quantum Electron. 26, 550 (1990).

9. C. R. Menyuk, IEEE J. Quantum Electron. 25, 2674 (1989).

10. N. J. Smith, K. J. Blow, and I. Andonovic, J. Lightwave Technol. 10, 1329 (1992).

11. A. Ruehl, D. Wandt, U. Morgner, and D. Kracht, Opt. Express 16, 8181 (2008). 